Predição de Propriedades Físicas de Ésteres Puros para a Modelagem da Combustão de Biodieseis

\author{
Arvelos, S.; Romanielo, L. L.
}

Rev. Virtual Quim., 2018, 10 (5), 1355-1372 Data de publicação na Web: 30 de outubro de 2018

http://rvq.sbq.org.br

\title{
Prediction the Physical Properties of Pure Esters for Modeling Biodiesel Combustion
}

Abstract: Adequate knowledge of biodiesel's physical properties is necessary to simulate its dispersion, atomization and combustion when it is being used in engines. The objective of this work was to evaluate predictive models of physical properties of seven main methyl and ethyl esters that compose biodiesel to model its combustion. The properties evaluated in this study were: normal boiling temperature, critical temperature, critical pressure, critical volume, acentric factor, saturation pressure, liquid density, enthalpy of vaporization and combustion, liquid viscosity, and surface tension. Satisfactory adjustments were obtained for each property evaluated by comparing estimated values to experimental data available in the literature.

Keywords: Biodiesel; esters; physical properties; combustion modeling.

\section{Resumo}

O conhecimento adequado das propriedades físicas de biodieseis é necessário para simular sua dispersão, atomização e combustão quando este combustível está sendo utilizado em motores. O objetivo deste trabalho foi avaliar modelos de predição de propriedades físicas dos sete principais ésteres metílicos e etílicos que compõem o biodiesel para modelagem da sua combustão. As propriedades avaliadas neste estudo foram: temperatura normal de ebulição, temperatura crítica, pressão crítica, volume crítico, fator acêntrico, pressão de saturação, densidade de líquidos, entalpias de vaporização e combustão, viscosidade de líquidos e tensão superficial. Foram obtidos ajustes satisfatórios para cada propriedade avaliada ao se comparar os valores estimados e aos experimentais disponíveis na literatura.

Palavras-chave: Biodiesel; ésteres; propriedades físicas; modelagem da combustão.

\footnotetext{
* Universidade Federal de Uberlândia, Faculdade de Engenharia Química, Av. João Naves de Ávila 2121, CEP 38408-144, Uberlândia-MG, Brasil.

Msarvelos@gmail.com DOI: $\underline{10.21577 / 1984-6835.20180093}$
} 


\section{Predição de Propriedades Físicas de Ésteres Puros para a Modelagem da Combustão de Biodieseis Sarah Arvelos, ${ }^{*}$ Lucienne L. Romanielo}

Universidade Federal de Uberlândia, Faculdade de Engenharia Química, Av. João Naves de Ávila 2121, CEP 38408-144, Uberlândia-MG, Brasil.

* sarvelos@gmail.com

Recebido em 24 de abril de 2018. Aceito para publicação em 22 de outubro de 2018

\section{Introdução}

\section{Modelos para Predição de Propriedades}

\section{Resultados e Discussão}

3.1. Temperatura normal de ebulição

3.2. Parâmetros críticos

3.3. Fator acêntrico

3.4. Capacidade calorífica de gases

3.5. Entalpia de vaporização

3.6. Entalpia de combustão

3.7. Viscosidade de líquidos

3.8. Tensão superficial

\section{Conclusão}

\section{Introdução}

O sistema de transportes exerce um importante papel no desenvolvimento da economia de qualquer país. Atualmente, a questão chave para o setor de transporte mundial é o suprimento de energia, que nas últimas décadas esteve sendo provido majoritariamente por combustíveis fósseis. Globalmente, segundo a U. S. Energy Information Administration, o consumo médio de energia no setor de transportes tem aumentado em $1,1 \%$ ao ano devido ao desenvolvimento da indústria automobilística. Estima-se que o setor de transportes terá uma participação de $63 \%$ no aumento do consumo global de combustíveis líquidos entre 2010 e 2040. Além disso, o crescimento significativo da indústria automobilística mundial resultou no aumento das emissões de poluentes. Atualmente, $22 \%$ das emissões globais de gases de efeito estufa vêm do setor de transportes. Estima-se que 8,6 bilhões de toneladas de $\mathrm{CO}_{2}$ serão liberados na atmosfera de 2020 a $2035 .{ }^{1}$

Nas últimas duas décadas, vários centros de pesquisa de todo o mundo vêm tentando descobrir e desenvolver tecnologias para a produção de combustíveis que sejam provenientes de fontes renováveis ou mais 
limpas em virtude de uma possível escassez do petróleo e crise ambiental. Dentre estas fontes de energia, o biodiesel é considerado o de maior viabilidade para substituição parcial ou total do diesel. O uso do biodiesel já é uma realidade em vários países do mundo, sendo empregado não só em veículo leves, mas também em veículos pesados e operação de máquinas industriais. $^{2}$

Biodieseis são misturas de mono-alquil ésteres de ácidos graxos de cadeia longa, provenientes predominantemente de óleos vegetais e gordura animal. ${ }^{3-4} A$ reação química que efetivamente está relacionada à transformação de óleos ou gorduras em biodiesel é conhecida como reação de transesterificação. A reação global de transesterificação consiste na reação entre um triglicerídeo e um álcool, com formação de um éster e outro álcool (glicerol), conforme representado na Figura 1. Nesta Figura, $R_{1}-R_{4}$ representam substituintes orgânicos. Metanol e etanol são os álcoois mais utilizados como reagentes e geram nesta reação ésteres metílicos e etílicos, respectivamente. ${ }^{5}$

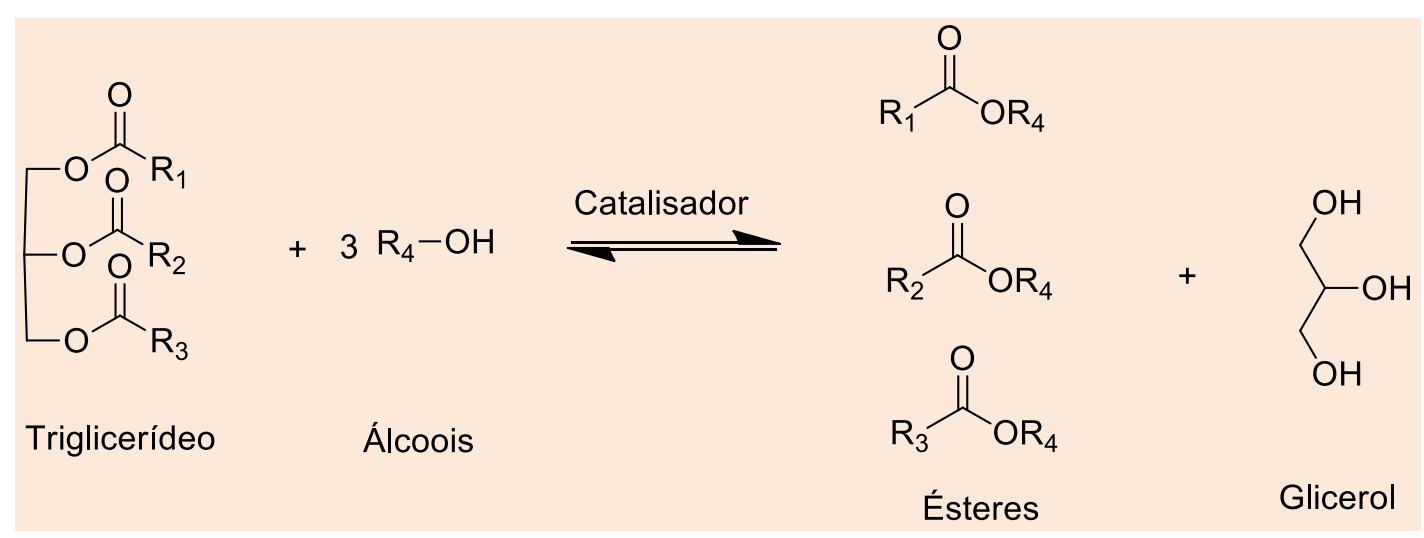

Figura 1. Esquema da reação geral de transesterificação

A transesterificação de óleos vegetais de soja, dendê, algodão, milho, sebo bovino e outras matérias-primas comumente utilizadas gera um biodiesel rico em ésteres de ácidos graxos do tipo láurico, mirístico, palmítico, esteárico, oleico, linoleico e linolênico. ${ }^{4}$ Logo, para se conhecer as propriedades do biodiesel, faz-se necessário o conhecimento das propriedades destes ésteres quando puros. Através de regras de mistura simples pode-se conhecer o comportamento da mistura final. ${ }^{6}$

Com estrutura molecular diferente do diesel comum, as propriedades físicas e químicas do biodiesel se diferem deste combustível fóssil. Logo, modelar a combustão do biodiesel é importante para conhecer o comportamento real deste combustível quando atomizado e evaporado. Além disso, essa modelagem é importante para se descrever a própria combustão desta mistura e a possível emissão de gases para a atmosfera. Apesar de certa quantidade de dados experimentais de propriedades de ésteres e biodiesel disponíveis na literatura, estas medidas ainda não estão disponíveis em grandes intervalos de temperatura e pressão. $^{7-9}$

Por causa disso, a busca por modelos eficientes para a previsão de propriedades de biodieseis tem sido tema de diversos trabalhos reportados na literatura. Tais trabalhos utilizaram diferentes metodologias para a previsão de propriedades, como equações de estado, redes neurais, métodos de contribuição de grupos e modelos empíricos. ${ }^{8-18}$ Dentre estes diversos métodos, destacam-se as duas últimas metodologias. Apesar de haver uma literatura disponível a respeito, as conclusões de cada trabalho dependem das substâncias avaliadas e da fonte dos dados experimentais consultados. 
Nem sempre todos os ésteres majoritários na composição do biodiesel foram estudados e nem todas as propriedades de interesse na modelagem da combustão foram contabilizadas.

O objetivo do presente trabalho foi realizar a predição de propriedades físicas e químicas de sete importantes ésteres que compõem os biodieseis metílicos e etílicos, contendo de 12 a 18 carbonos: láurico, mirístico, palmítico, esteárico, oleico, linoleico e linolênico. As propriedades avaliadas neste estudo foram: temperatura normal de ebulição, temperatura crítica, pressão crítica, volume crítico, fator acêntrico, pressão de saturação, densidade de líquido, entalpias de vaporização e combustão, viscosidade absoluta de líquidos e tensão superficial.

Dentre as propriedades estudadas, as sete primeiras delas são importantes para a compreensão do processo de mistura e disposição de fases dos combustíveis no motor. Os parâmetros críticos, em especial, são fundamentais para o uso de equações de estado cúbicas, as quais têm sido as equações mais utilizadas na engenharia de processos químicos para a descrição de derivados do petróleo e biocombustíveis. ${ }^{19-20}$ De todas estas propriedades, as entalpias de vaporização e combustão auxiliam no cálculo do poder calorífico do biodiesel. O poder calorífico é um dos parâmetros mais influentes que afetam o consumo específico de combustível, eficiência térmica e emissões de NOx do motor. ${ }^{21}$ Já a viscosidade é um influente fator na atomização e na combustão dos biodieseis. A maior viscosidade do diesel comparada a do diesel tem sido um problema para o projeto de equipamentos. Se a viscosidade do biodiesel é alta, a atomização ocorre com gotículas maiores dificultando a combustão. Se a viscosidade é baixa, as gotículas formadas podem ser tão pequenas que há deficiência de lubrificação causando desgaste no motor e vazamentos. ${ }^{22}$ Assim como a viscosidade, a tensão superficial também é uma propriedade importante para a atomização de um combustível. Altos valores de tensão superficial dificultam a formação de gotas. ${ }^{23}$

\section{Modelos para Predição de Propriedades}

Para realização deste trabalho, foi necessária a identificação dos grupos funcionais que representam as moléculas de interesse, que são os ésteres metílicos e etílicos láurico, mirístico, palmítico, esteárico, oleico, linoleico e linolênico. A implementação de todos os modelos foi realizada em planilhas do software Excel 2007. Os valores estimados para cada propriedade de interesse foram comparados com valores experimentais disponíveis na literatura. Neste trabalho, a comparação entre os valores estimados e experimentais se deu através da média dos desvios médios relativos (DMR).

A Tabela 1 apresenta as propriedades selecionadas para avaliação neste trabalho e a referência bibliográfica dos modelos selecionados para predição destas propriedades. A descrição dos modelos e maiores detalhes sobre a metodologia utilizada serão apresentados em conjunto com os resultados do presente trabalho. 
Arvelos, S.; Romanielo, L. L.

Tabela 1. Propriedades avaliadas e referência dos métodos de estimação

\begin{tabular}{|c|c|}
\hline Propriedade & Métodos de predição e referência \\
\hline \multirow[t]{4}{*}{ Temperatura normal de ebulição $\left(T_{b}\right)$} & Joback e Reid - MJR; ${ }^{24}$ \\
\hline & Constantinou e Gani - MCG; ${ }^{25}$ \\
\hline & $\begin{array}{l}\text { Marrero-Marejon e Pardillo-Fontdevilla - } \\
\text { MMP; }{ }^{26}\end{array}$ \\
\hline & Marrero e Gani - MMG. ${ }^{27}$ \\
\hline \multirow{4}{*}{$\begin{array}{l}\text { Propriedades críticas: Temperatura }\left(T_{c}\right) \text {, } \\
\text { Pressão }\left(P_{c}\right) \text { e Volume }\left(V_{c}\right)\end{array}$} & Ambrose - MA; ${ }^{28}$ \\
\hline & MJR; \\
\hline & MCG; \\
\hline & MMG. \\
\hline \multirow[t]{5}{*}{ Fator acêntrico $(\omega)$} & Pitzer - RP; ${ }^{28}$ \\
\hline & Ambrose-Walton - RAW; ${ }^{28}$ \\
\hline & Kesler e Lee (1976) - RKL; ${ }^{29}$ \\
\hline & Edmister - RE; ${ }^{30}$ \\
\hline & Han e Peng - RHP. ${ }^{31}$ \\
\hline \multirow[t]{2}{*}{ Capacidade calorífica de gases ideais $\left(C_{p G}\right)$} & MJR; \\
\hline & Rihani e Doraiswamy - MRD. ${ }^{32}$ \\
\hline Capacidade calorífica de líquidos $\left(C_{p L}\right)$ & Bondi. $^{33}$ \\
\hline \multirow[t]{5}{*}{ Entalpia de vaporização ( $\left.\Delta H^{v a p}\right)$} & Reidel; $^{28}$ \\
\hline & Chen ${ }^{28}$; \\
\hline & Vetere; ${ }^{28}$ \\
\hline & Método de Watson; ${ }^{28}$ \\
\hline & MCG. \\
\hline \multirow{3}{*}{$\begin{array}{l}\text { Entalpia de combustão no estado padrão } \\
\qquad\left(H^{c}\right)\end{array}$} & MJR; \\
\hline & MCG; \\
\hline & MMG. \\
\hline \multirow[t]{2}{*}{ Viscosidade de líquidos $\left(\mu^{t}\right)$} & Letsou e Stiel; ${ }^{8}$ \\
\hline & Krisnangkura et al. ${ }^{34}$ \\
\hline \multirow[t]{2}{*}{ Tensão superficial $(\sigma)$} & Métodos de Brock e Bird; ${ }^{28}$ \\
\hline & Pitzer e Curl. ${ }^{28}$ \\
\hline
\end{tabular}




\section{Resultados e Discussão}

\subsection{Temperatura normal de ebulição}

As formulações dos métodos $\mathrm{MJR}^{24}$, $\mathrm{MCG}^{25}, \mathrm{MMP}^{26}$ e $\mathrm{MMG}^{27}$ são do tipo descrito na Equação 1, nas quais $T_{b}$ é a temperatura normal de ebulição e $N_{k}$ é o número de grupos $k$ com contribuição $T_{b k}$ que é dependente do método utilizado. $\beta$ e $\kappa$ são constantes. Os dados experimentais avaliados foram obtidos em Graboski e McCormick ${ }^{35}$ e Santander et al. ${ }^{36}$ para os ésteres metílicos laurato, palmitato, estearato, oleato, linoleato e etílicos palmitato, oleato e linoleato.

$$
T_{b}=\beta+\kappa \sum_{k} N_{k}\left(T_{b k}\right)
$$

A Figura 2 mostra o ajuste dos dados preditos aos dados experimentais. A linha reta mostrada nesta figura é a reta bissetriz do primeiro quadrante.

Os métodos MJR, MCG, MMP e MMG apresentaram as médias dos desvios médios relativos de 10,65; 2,92; 4,26 e 3,94 respectivamente. Considerando que o nível de confiança das medidas experimentais é de \pm 19
$\mathrm{K}^{36}$ os métodos MCG, MMP e MMG apresentam boas estimativas de temperatura normal de ebulição. O método de Constantinou e Gani (MCG) foi selecionado para estimativas posteriormente necessárias. Endossando essa escolha, ressalta-se que em trabalho recente realizado por Evangelista et al., ${ }^{37}$ foram apresentados indicativos de que o método MCG seja superior ao método MJR para avaliação de temperatura crítica.

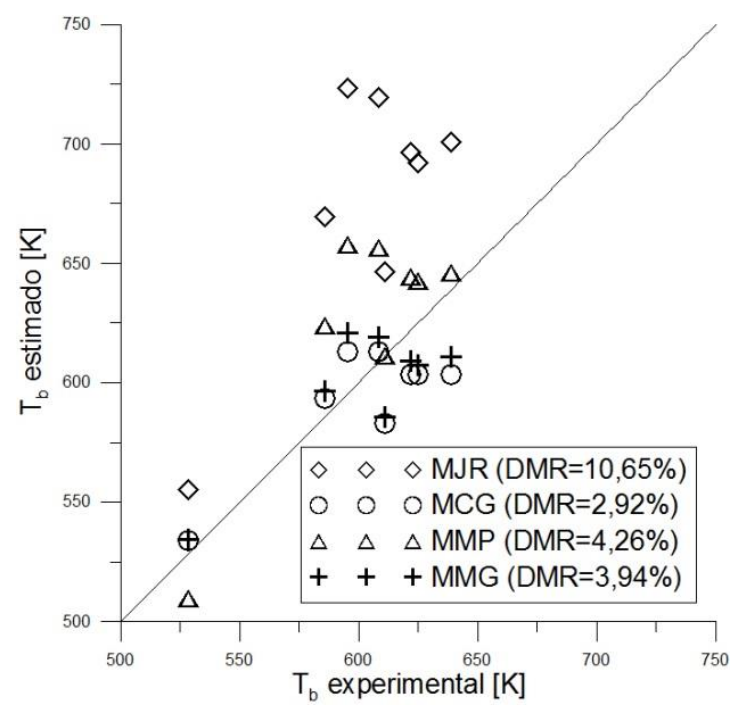

Figura 2. Médias dos desvios médios relativos (DMR's) para avaliação de dados de temperatura normal de ebulição $\left(T_{b}\right)$ obtidos experimentalmente e estimados via diversos métodos de contribuição de grupos 


\subsection{Parâmetros críticos}

Os modelos $\mathrm{MA},{ }^{28} \mathrm{MJR}^{24} \mathrm{MCG}^{25}$ e $\mathrm{MMG}^{27}$, os quais são métodos de contribuição de grupos, foram utilizados para estimativa de $T_{c}$, $P_{c}$ e $V_{c}$. Como para os ésteres de cadeia longa ainda não existem medidas experimentais para todos os tamanhos de cadeia e número de insaturações optou-se pela realização da previsão destas propriedades.

Para avaliação de $T_{c}$ e $P_{c}$ preditos, quanto a sua adequabilidade, dados experimentais de pressão de saturação $\left(P^{v p}\right)$ e densidade de líquidos $\left(\rho^{\text {liq }}\right)$ foram selecionados da literatura. ${ }^{18,38-45}$ Estes dados foram utilizados para regressão de $T_{c}, P_{c}$ e $\omega$ pela equação de Peng e Robinson ${ }^{46}$ com o software PE2000, fazendo uso do método simplex com a função objetivo $\left(F_{o b j}\right)$ representada pela Equação (2). Nesta Equação, $N_{p}$ representa o número de pontos experimentais e os subscritos exp e calc representam os valores experimentais e calculados, respectivamente.

$$
F_{o b j}=100 \%\left[\sum_{i=1}^{N p}\left|\left(\frac{\rho_{\exp }^{l i q}-\rho_{c a l c}^{l i q}}{\rho_{\exp }^{l i q}}\right)\right|+\sum_{i=1}^{N p}\left|\left(\frac{P_{\exp }^{v p}-\rho_{c a l c}^{v p}}{P_{\exp }^{v p}}\right)\right|\right]
$$

A equação de Peng e Robinson apresentou bons ajustes aos dados experimentais de pressão de saturação e densidade de líquidos. Para pressão de saturação, o menor desvio obtido foi referente ao metil palmitato $(0,21$ $\%)$ e o maior foi para o etil palmitato (1,79 \%). Para a densidade, o menor desvio foi para o etil palmitato $(2,04 \%)$ e o maior para o metil estearato $(2,71 \%)$. No trabalho de Pratas et al. ${ }^{18}$, o máximo valor obtido para o desvio padrão na medida de densidade foi de $0,15 \%$. Segundo Silva et al. ${ }^{45}$ e o banco de dados do Design Institute for Physical Properties $(D I P P R)^{47}$ os desvios entre medidas experimentais de pressão de saturação de ésteres estão em torno de 3,5 \% a $5 \%$. Então, julga-se que os desvios obtidos pelo ajuste dos parâmetros à equação de Peng e Robinson foram inferiores aos valores experimentais e esta equação de estado se mostra boa ferramenta para avaliação de densidade de líquidos e pressão de saturação das substâncias avaliadas.

Para endossar o uso destas propriedades como meio de validação, cita-se o trabalho de Nikitin et al. ${ }^{48}$ Tais autores realizaram a medida experimental da temperatura crítica dos ésteres metil palmitato e metil oleato. Os valores obtidos foram de $760 \pm 8 \mathrm{~K}$ e $785 \pm 8 \mathrm{~K}$, respectivamente. Tais valores estão de acordo com os obtidos via regressão que foram de 766,20 e $772,87 \mathrm{~K}$.

Comparando os valores obtidos via regressão de $T_{c}$ e $P_{c}$ com os valores estimados pelos métodos de contribuição de grupos, nota-se que o método de Marrero-Gani (MMG) obteve os menores desvios em relação a estas duas propriedades. Para a temperatura crítica, a média dos desvios médios relativos foi $1,16 \%$ e para a pressão crítica a média dos desvios médios relativos foi de $9,97 \%$. A comparação entre $T_{c}$ e $P_{c}$ obtidos via regressão com os valores calculados via métodos de contribuição de grupos pode ser feita pela análise da Figura 3. Na Figura 3(a) apresenta-se os resultados de $T_{c}$ e na Figura 3(b) apresenta-se os dados de $P_{c}$.

Para avaliação do melhor método para estimativa do volume crítico, foi considerado o fator de compressibilidade crítico $\left(Z_{c}\right)$ da equação de Peng e Robinson de 0,3074 e, assim, pode-se calcular o volume crítico ajustado via $T_{c}$ e $P_{c}$ ajustados. Os quatro métodos de contribuição de grupos fizeram estimativas próximas entre si. 0 menor valor estimado de $V_{c}$ foi referente ao metil laurato $\left(V_{c}=781,2 \mathrm{~cm}^{3} / \mathrm{mol}\right.$ pelo $\left.\mathrm{MA}\right)$ e o maior valor estimado foi referente ao etil estearato $\left(V_{c}=1158,45 \mathrm{~cm}^{3} / \mathrm{mol}\right.$ pelo MMG). 0 método 
que obteve os menores desvios médios relativos foi o MCG, com média geral de 18,81 \%. MA, MJR e MMG obtiveram média dos

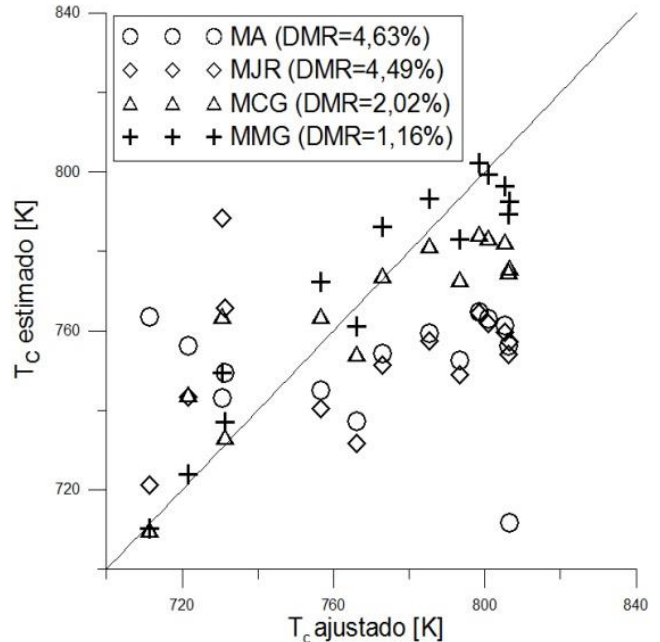

(a) desvios médios relativos de 21,23; 20,27 e $19,86 \%$, respectivamente.

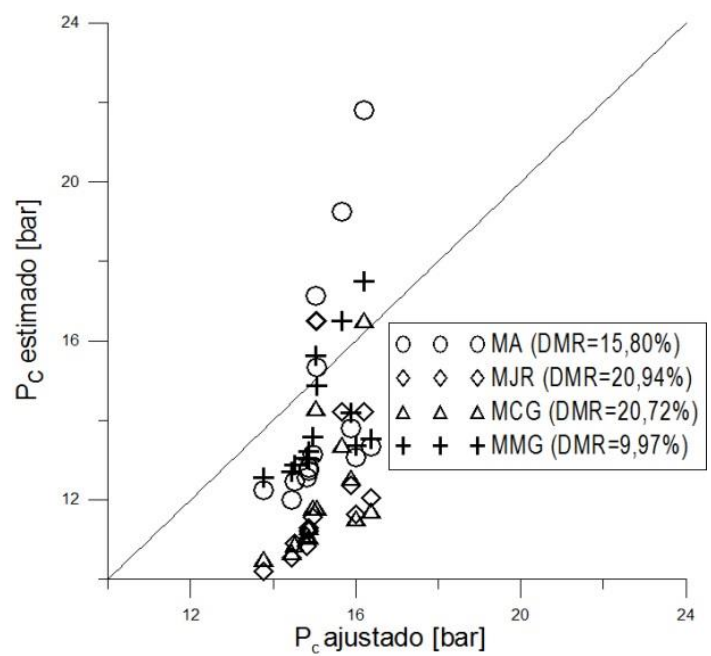

(b)

Figura 3. Médias dos desvios médios relativos (DMR's) para avaliação de dados de temperatura crítica $\left(T_{c}\right)$ e pressão crítica $\left(P_{c}\right)$ ajustados à equação de Peng e Robinson e estimados via diversos métodos de contribuição de grupos

A incerteza na medida do volume crítico do éster metílico de 4 carbonos (metil butanoato) está em torno de $5 \%$, segundo o DIPPR. Considerando as incertezas nos cálculos provenientes do uso sucessivo de estimativas (regressão de $T_{c}$ e $P_{c}$, fixação do fator de compressibilidade crítico) e a diferença no tamanho da cadeia de um éster C4 (com quatro carbonos) para ésteres $\mathrm{C} 12$ - C18, pode-se dizer que o método de Constantinou-
Gani apresenta-se como uma ferramenta razoável na estimativa de volume crítico de ésteres de cadeia longa.

\subsection{Fator acêntrico}

Os modelos avaliados neste trabalho são apresentados a seguir:

- Regra de Pitzer (RP): ${ }^{28} Z_{c}=0,291-0,080 \omega$

- Regra de Kesler e Lee (RKL): ${ }^{29} \omega=\frac{-\ln P_{c}-5,93+6,10 / T_{b r}+1,29 \ln T_{b r}-0,17 T_{b r}^{6}}{15,25-15,687 / T_{b r}-13,47 \ln T_{b r}+0,44 T_{b r}^{6}}$ - Regra de Ambrose e Walton (RAW): ${ }^{28}$ É
um modelo baseado nos estados
correspondentes e utiliza dois parâmetros. $T_{b r}$ representa a temperatura reduzida e $f^{(0)}$ e $f^{(1)}$ são funções da temperatura normal de ebulição reduzida: 


$$
\omega=\left\lfloor-\ln \left(P_{c} / 1,01325\right)+f^{(0)}\right\rfloor / f^{(1)}
$$

$$
\text { - Regra de Edmister (RE): }{ }^{30} \omega=\left[\frac{3}{7}\left(\frac{T_{b} / T_{c}}{1-T_{b} / T_{c}}\right) \log \left(\frac{P_{c}}{P_{a t m}}\right)\right]
$$

- Regra de Han e Peng (RHP): ${ }^{31}$ estima o fator acêntrico através de contribuição de grupos.

Os valores estimados de fator acêntrico para os 14 ésteres de interesse foram comparados aos obtidos no ajuste de densidade e pressão de saturação à equação de Peng e Robinson. A Figura 4 mostra o ajuste de dados. A melhor regra para previsão de fator acêntrico foi a regra de Han e Peng (RHP) com desvio médio relativo de 7,02 \%. Uma explicação plausível para o sucesso da RHP se baseia no fato de que as estimativas realizadas dependeram unicamente da estrutura das moléculas. Demais métodos (RP, RKL, RAW e
RE) dependeram de outras estimativas, como, por exemplo, $T_{b}, Z_{c}, T_{c}$, e podem trazer consigo o erro acumulado da estimativa destas propriedades.

Selecionada a combinação de parâmetros $T_{c}$ por MMG, $P_{c}$ por MMG e $\omega$ pela RHP obtémse, recalculando pressão de saturação e densidade de líquidos, desvios médios de $24,37 \%$ e $15,84 \%$, respectivamente; o que mostra que esta combinação de parâmetros oferece uma boa estimativa de densidade e de pressão de saturação. Porém, novos estudos devem ser realizados, visando buscar métodos que calculem densidade e pressão de saturação dentro das incertezas das medidas.

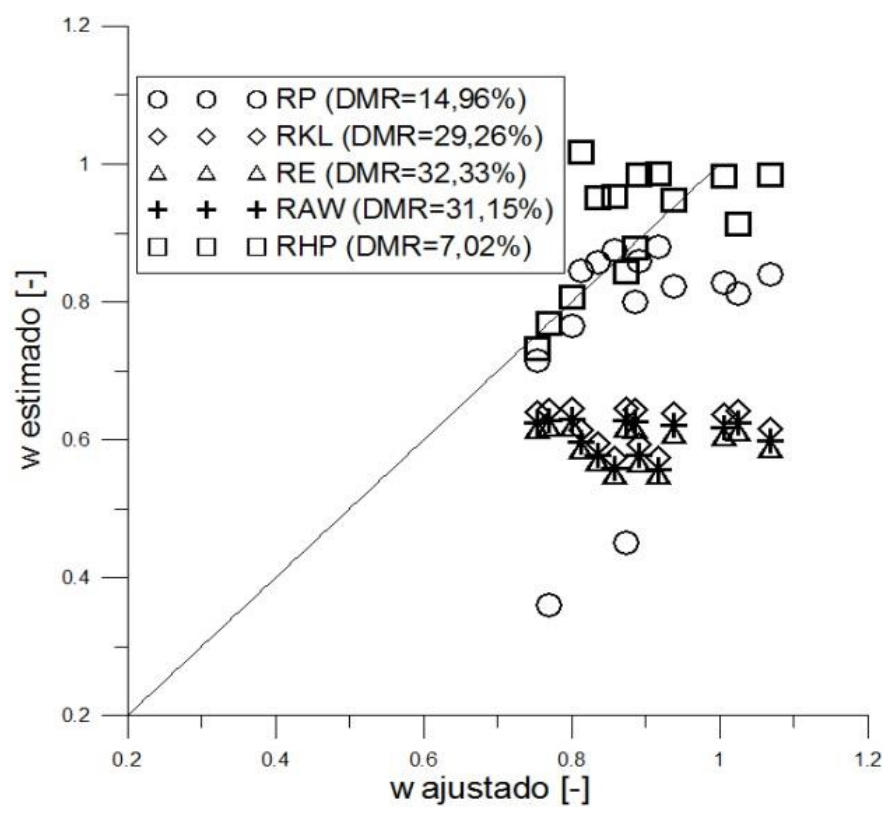

Figura 4. Médias dos desvios médios relativos (DMR's) para avaliação de dados de fator acêntrico $(\omega)$ ajustados à equação de Peng e Robinson e estimados via diversos métodos 


\subsection{Capacidade calorífica de gases}

Para a avaliação de um melhor método apto para cálculo da capacidade calorífica, na ausência de dados experimentais para gases, utilizou-se uma equação de predição de capacidade calorífica de líquidos, $C_{p L}$, em conjunto com a equação apresentada em Bondi: ${ }^{33}$

$C_{p L}-C_{p G}=R\left\{1,45+0,45\left(1-T_{r}\right)^{-1}+0,25 \omega\left[17,11+25,2\left(1-T_{r}\right)^{\frac{1}{3}} T_{r}+1,742\left(1-T_{r}\right)^{-1}\right]\right\}(7)$

Os métodos de contribuição de grupos de Joback e Reid (MJR) ${ }^{24}$ e Rihani e Doraiswamy $(M J R)^{32}$ foram utilizados para calcular a capacidade calorífica do gás ideal.

Foram obtidos na literatura dados de capacidade calorífica do líquido para metil laurato, miristato, palmitato e estearato. ${ }^{49}$ Nas medidas realizadas na literatura citada, as incertezas foram inferiores a $3 \%$. Os métodos MJR e MRD apresentaram estimativas bastante próximas. Sendo que o MJR apresentou média dos desvios médios relativos de 2,14 \% e o MRD apresentou de 3,1 $\%$. A Figura 5 mostra o ajuste para os ésteres metílicos palmitato e estearato.

Ceriani et al. ${ }^{50}$ utilizaram o modelo de Bondi em conjunto com o MJR para o cálculo da capacidade calorífica de alguns triglicerídeos (trilaurina, trimiristina, tripalmitina e tristearina) e diversos ésteres. Para os ésteres observaram desvio médio de $8,49 \%$, os quais são resultados equivalentes aos obtidos neste trabalho.
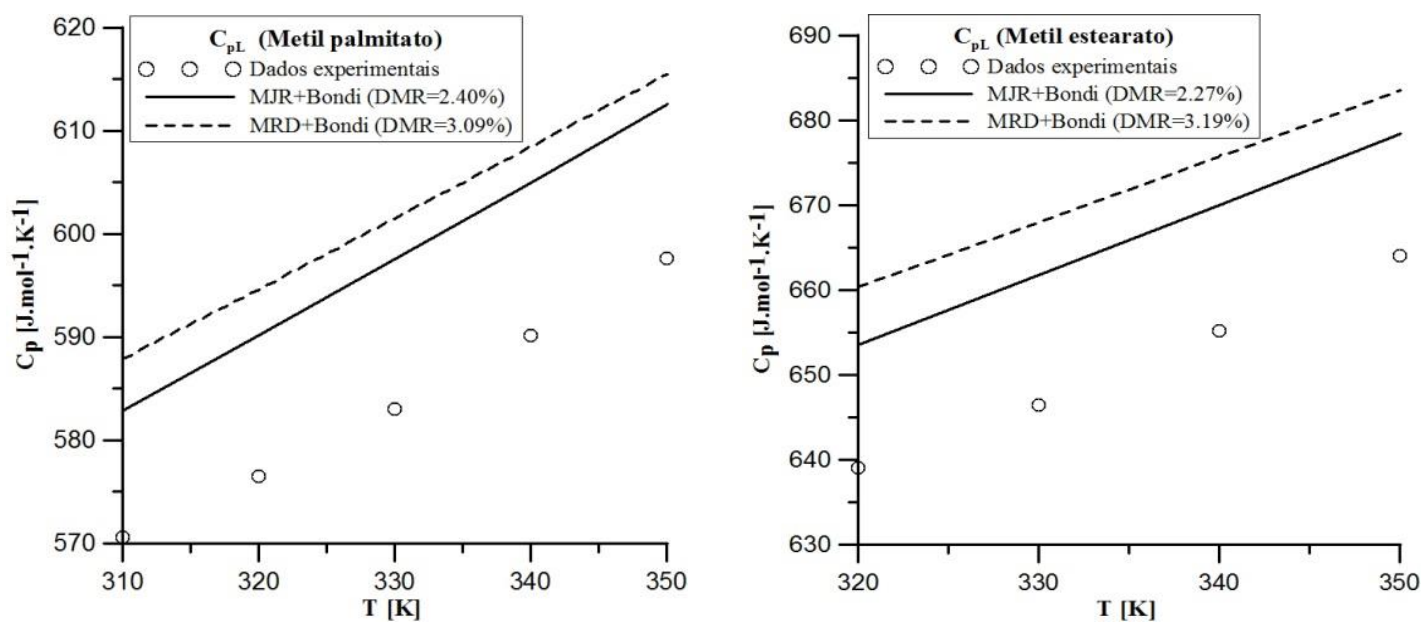

Figura 5. Correlação dos dados experimentais de capacidade calorífica de metil palmitato e metil estearato líquidos $\left(C_{p L}\right)$ utilizando os modelos MJR e MRD modificados pelo modelo de Bondi

\subsection{Entalpia de vaporização}

Os métodos de Reidel, Chen e Vetere ${ }^{28}$ se baseiam na equação de Clayperon e calculam a entalpia de vaporização estando o fluido na temperatura normal de ebulição. O método de Constantinou e Gani (MCG) ${ }^{25}$ estima a entalpia de vaporização pela metodologia de 
contribuição de grupos, contudo a estimativa é realizada a $298 \mathrm{~K}$. Tais métodos (Reidel, Chen, Vetere e MCG) foram utilizados para calcular a entalpia de vaporização dos ésteres em conjunto com o método de Watson..$^{28}$ Este método avalia a entalpia de vaporização em uma dada temperatura tendo um valor referencial de entalpia de vaporização conhecido. $\mathrm{O}$ uso do método de Watson se fez necessário para corrigir os valores estimados, uma vez que as medidas experimentais consultadas estavam em temperaturas variadas. Os modelos de Vetere e Watson são representados pelas Equações (8) e (9):

- Método de Vetere: $H_{b}^{v a p}=R T_{b} \frac{\left(1-T_{b r}\right)^{0,38}\left(\ln P_{c}-0,513+0,5066 /\left(P_{c} T_{b r}^{2}\right)\right)}{1-T_{b r}+\left(1-\left(1-T_{b r}\right)^{0,38}\right) \ln T_{b r}}$

-Método de Watson: $H_{2}^{v a p}=\Delta H_{1}^{v a p}\left(\frac{1-T_{r 2}}{1-T_{r 1}}\right)^{0,38}$

Para avaliação da modelagem, dados experimentais de entalpia de vaporização de metil laurato e metil miristato; ${ }^{51}$ de metil palmitato, estearato, oleato, linoleato e linolenato ${ }^{52}$ e de etil palmitato, estearato, oleato e linoleato ${ }^{45}$ foram utilizados. Medidas experimentais de entalpia de vaporização possuem incertezas variando entre $5-10 \%$, segundo o DIPPR. A Figura 6 apresenta os resultados obtidos para entalpia de vaporização. Nota-se que o menor erro obtido foi para o MCG, com média dos desvios médios relativos de $9,56 \%$. Este método foi selecionado para cálculos posteriores.

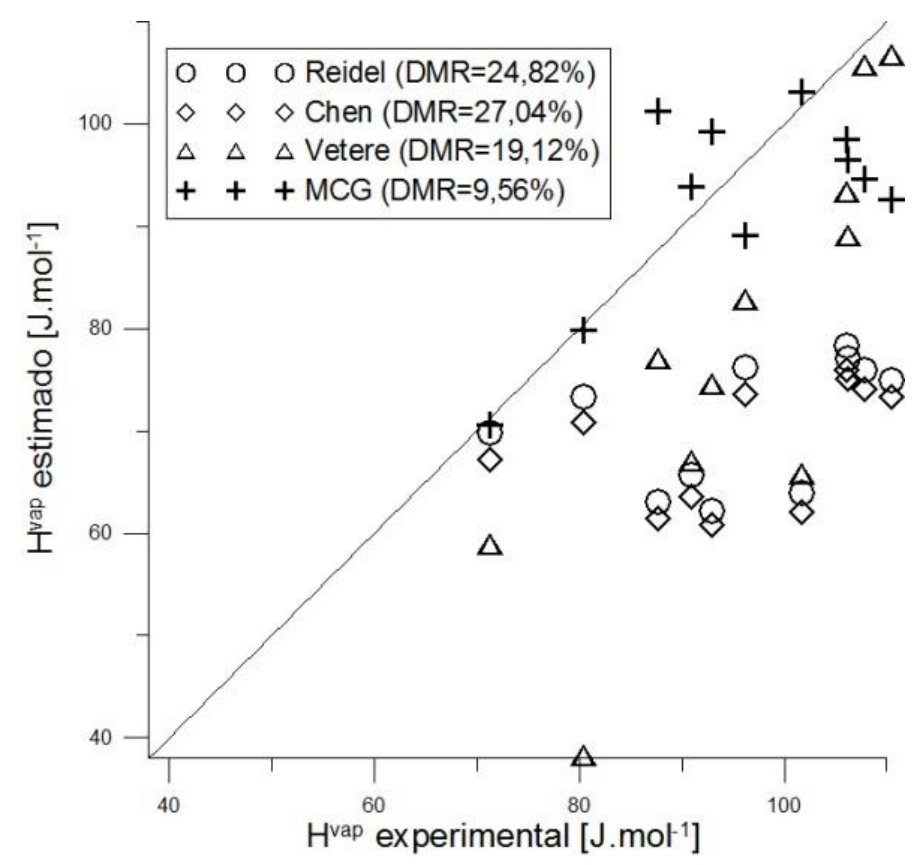

Figura 6. Médias dos desvios médios relativos (DMR's) para avaliação de dados de entalpia de vaporização estimados pelos métodos de Reidel, Chen, Vetere e MCG 


\subsection{Entalpia de combustão}

Nos cálculos efetuados neste trabalho, os dados experimentais de entalpia de combustão foram calculados a partir dos dados estimados de entalpia de formação dos gases, avaliados pelos métodos de Joback-

$$
H_{f}^{0}=\alpha+\sum_{k} N_{k}\left(H_{f k}\right)
$$

Os dados experimentais avaliados de entalpia de combustão estão disponíveis em Freedman e Bagby. ${ }^{53}$ A Figura 7 apresenta os resultados obtidos para entalpia combustão. Os três métodos avaliados apresentaram excelentes predições, obtendo média dos desvios médios relativos menores que $1 \%$ que é o valor da incerteza da medida reportada pelos autores. Os erros logrados são da
Reid (MJR), Constantinou-Gani (MCG) e Marrero-Gani (MMG). Nestes métodos, a entalpia de formação é estimada a partir de uma relação linear entre o número de grupos $\left(N_{k}\right)$ e a contribuição de grupos funcionais $\left(H_{f k}\right)$, como na Equação (10), na qual $\alpha$ é uma constante:

mesma ordem de grandeza dos obtidos por Mehta e Anand, ${ }^{54}$ os quais propuseram um método para previsão do poder calorífico de biodieseis que é baseado nas energias de ligação das moléculas constituintes dos biodieseis. Para 15 diferentes biodieseis, os erros das estimativas realizadas quando comparados com dados experimentais estiveram próximos a $3 \%$.

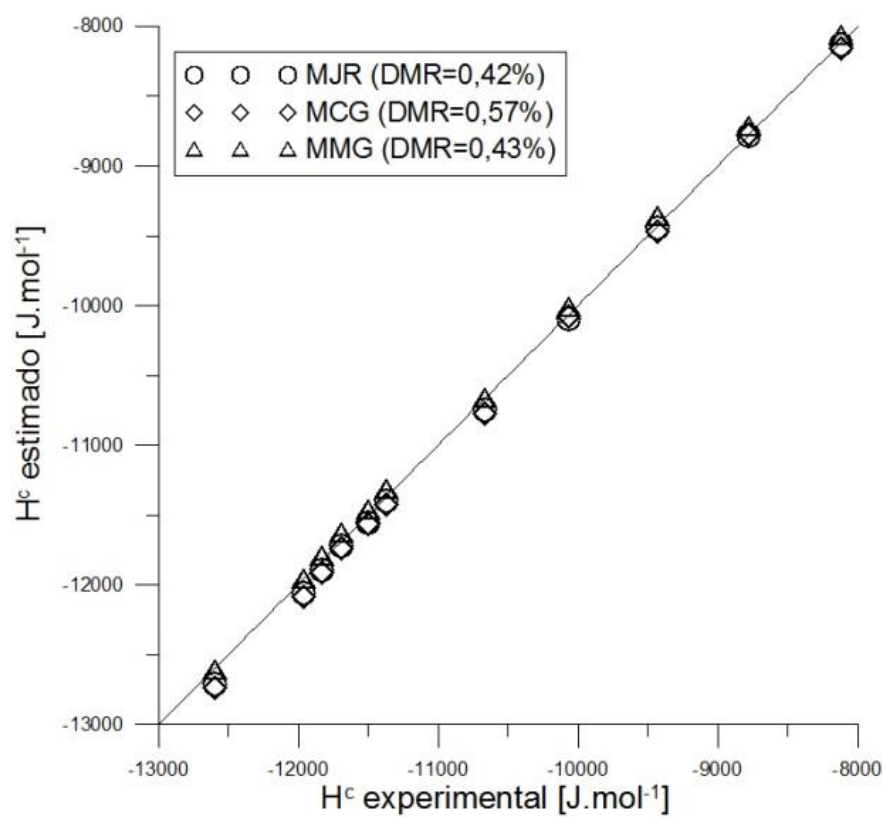

Figura 7. Médias dos desvios médios relativos (DMR's) para avaliação de dados de entalpia de combustão estimados pelos métodos MJR, MGC e MCG 


\subsection{Viscosidade absoluta de líquidos}

Nesta seção, os métodos avaliados foram os de Letsou e Stiel ${ }^{8}$ e de Krisnangkura et al. ${ }^{34}$ O primeiro método é representado por um modelo empírico como na Equação (11), na qual $(\mu \xi)^{(0)}$ e $(\mu \xi)^{(1)}$ são funções da temperatura reduzida e $\xi$ é uma função de $T_{c}$, $P_{c}$ e massa molar da substância. A Equação (11) foi desenvolvida para previsão de

$$
\mu^{L} \xi=(\mu \xi)^{(0)}+\omega(\mu \xi)^{(1)}
$$

A Tabela 2 mostra a eficiência do ajuste dos valores estimados aos dados experimentais de viscosidade absoluta disponíveis no banco de dados DIPPR. Esta Tabela mostra também o número de dados experimentais disponíveis $\left(N_{p}\right)$ e o intervalo de temperatura avaliado. Como o modelo de Krisnangkura et al. ${ }^{9}$ estima valores de viscosidade cinemática, multiplicou-se os valores preditos de viscosidade pela densidade calculada dos ésteres para obtenção da viscosidade absoluta.

Pelos resultados apresentados na Tabela 2 , nota-se que o modelo de Letsou e Stiel ${ }^{8}$ foi inadequado para a representação da viscosidade de biodieseis, uma vez que as incertezas máximas nas medidas estão em torno de $25 \%$ para o metil linolenato. Uma explicação plausível para este resultado reside no fato de que tal modelo foi obtido para substâncias de natureza química e tamanho de cadeia bem diferentes dos ésteres avaliados neste trabalho. An et al. ${ }^{9}$ avaliaram a capacidade preditiva deste modelo para previsão da viscosidade do metil oleato em viscosidade absoluta de líquidos não-polares, tais como alcanos e gases nobres. No segundo método, considerando o número de carbonos, os autores propuseram equações para determinação da viscosidade cinemática de biodieseis em diferentes temperaturas. Para obtenção da modelagem, os autores regrediram os parâmetros das equações empíricas ajustando dados experimentais de ésteres metílicos de cadeias dotadas de 4 a 18 carbonos, em intervalo de temperatura de 293 a $353 \mathrm{~K}$.

intervalo de temperatura de 300 a $600 \mathrm{~K}$. Assim como no presente trabalho, os autores obtiveram erros elevados na modelagem de 300 a $400 \mathrm{~K}$. Acima de $400 \mathrm{~K}$ o ajuste foi razoável, o que pode ser atribuído à diminuição das forças intermoleculares no interior do fluido, fazendo com que o comportamento da viscosidade se assemelhe ao de moléculas apolares.

O modelo de Krisnangkura et al. $^{34}$ apresentou erros dentro do intervalo de incerteza da medida para a maioria dos ésteres. Erros elevados foram obtidos para metil esterearato, etil palmitato, etil estearato, etil linoleato e etil linolenato, conforme pode ver visualizado na Tabela 2 . Erros acima de $25 \%$ ocorreram quando as temperaturas avaliadas excederam as de 453 , $453,422,477$ e $491 \mathrm{~K}$ para os cinco ésteres citados, respectivamente. Tais temperaturas estão bem acima das utilizadas na obtenção do modelo de Krisnangkura et al., ${ }^{34}$ o que indica que o modelo apresenta um limite de segurança ao ser extrapolado. 
Tabela 2. Número de pontos experimentais $\left(N_{p}\right)$, intervalo de temperatura e ajuste de viscosidade de líquidos aos métodos de estimação

\begin{tabular}{ccccc}
\hline \multirow{2}{*}{ Éster } & $\boldsymbol{N}_{\boldsymbol{p}}$ & $\begin{array}{c}\text { Intervalo de } \\
\text { temperatura [K] }\end{array}$ & \multicolumn{2}{c}{ DMR [\%] (mínimo-máximo) } \\
\cline { 5 - 5 } & & & Letsou e Stiel ${ }^{8}$ & Krisnangkura et al. $^{34}$ \\
\hline Metil laurato & 9 & $293-372$ & $65,03(44,81-79,29)$ & $5,99(0,2-8,40)$ \\
Metil miristato & 10 & $293-372$ & $72,20(52,06-84,89)$ & $2,55(0,40-5,16)$ \\
Metil palmitato & 5 & $310-372$ & $72,89(58,33-82,15)$ & $6,94(3,75-6,92)$ \\
Metil estearato & 8 & $313-573$ & $54,19(11,39-84,74)$ & $22,08(8,31-47,00)$ \\
Metil oleato & 3 & $303-363$ & $71,71(59,73-83,41)$ & $2,90(1,04-5,03)$ \\
Metil linoleato & 7 & $283-343$ & $71,85(58,15-84,50)$ & $14,29(11,88-17,24)$ \\
Metil linolenato & 9 & $283-363$ & $53,84(26,80-80,53)$ & $20,69(7,68-32,60)$ \\
Etil laurato & 15 & $273-368$ & $72,71(47,28-87-79)$ & $3,34(0,39-12,00)$ \\
Etil miristato & 12 & $293-394$ & $68,24(44,36-84,61)$ & $5,74(0,60-9,94)$ \\
Etil palmitato & 13 & $298-573$ & $59,96(5,52-86,95)$ & $13,43(0,08-46,73)$ \\
Etil estearato & 15 & $308-573$ & $56,51(8,43-86,00)$ & $20,19(0,11-55,11)$ \\
Etil oleato & 8 & $293-373$ & $75,98(55,48-87,44)$ & $8,43(5,78-13,82)$ \\
Etil linoleato & 31 & $218-628$ & $58,79(11,58-98,67)$ & $17,44(0,05-53,14)$ \\
Etil linolenato & 32 & $256-628$ & $53,54(12,97-91,23)$ & $14,96(0,31-49,27)$ \\
\hline
\end{tabular}

\subsection{Tensão superficial}

O método de Brock e Bird ${ }^{28}$ é representado pela Equação (12) e o método de Ptizer e
Curl ${ }^{28}$ pela Equação (13). Ambos os modelos são baseados no Teorema dos Estados Correspondentes.

$$
\begin{gathered}
\sigma=P_{c}^{2 / 3} T_{c}^{1 / 3}\left\{0,132\left\{0,9076\left[1+\frac{T_{b r} \ln \left(P_{c}-1,01325\right)}{1-T_{b r}}\right]\right\}-0,279\right\}\left(1-T_{r}\right)^{1 / 9} \\
\sigma=P_{c}^{2 / 3} T_{c}^{1 / 3} \frac{1,86+1,18 \omega}{19,05}\left[\frac{3,75+0,91 \omega}{0,291-0,08 \omega}\right]^{2 / 3}
\end{gathered}
$$

Os dados experimentais de tensão superficial obtidos na literatura para os ésteres de interesse são os disponíveis no banco de dados DIPPR. A Tabela 3 mostra a ajuste dos dados experimentais aos dados preditos, o número de dados experimentais disponíveis $\left(N_{p}\right)$ e o intervalo de temperatura avaliado. Para os dados experimentais de tensão superficial as incertezas na medida estão entre 1 e $5 \%$. Sendo, então, o método de Pitzer e Curl efetivo para cálculo desta propriedade, para maioria dos ésteres. Em trabalho disponível na literatura, ${ }^{9}$ o método de Brock e Bird ${ }^{28}$ foi indicado para a previsão 
da tensão de superficial de ésteres metílicos de cadeia longa. $O$ presente trabalho mostra que o modelo Ptizer e $\mathrm{Cur}^{28}$ se apresenta mais adequado que o primeiro modelo, tanto para ésteres metílicos quanto etílicos.

Tabela 3. Número de pontos experimentais $\left(N_{p}\right)$, intervalo de temperatura e ajuste de tensão superficial de líquidos aos métodos de estimação

\begin{tabular}{ccccc}
\hline Éster & $\boldsymbol{N}_{\boldsymbol{p}}$ & $\begin{array}{c}\text { Intervalo de } \\
\text { temperatura [K] }\end{array}$ & \multicolumn{2}{c}{ DMR [\%] (mínimo-máximo) } \\
\cline { 5 - 5 } & & & Brock e Bird $^{\mathbf{2 8}}$ & Pitzer e Curl $^{\mathbf{2 8}}$ \\
\hline Metil laurato & 11 & $286-360$ & $5,88(6,42-7,29)$ & $1,29(0,84-2,08)$ \\
Metil miristato & 10 & $293-373$ & $9,22(9,02-9,30)$ & $1,94(1,86-2,17)$ \\
Metil palmitato & 10 & $303-373$ & $13,63(13,42-13,67)$ & $3,20(2,99-3,45)$ \\
Metil estearato & 8 & $313-348$ & $17,69(16,89-18,07)$ & $4,55(4,08-5,56)$ \\
Metil oleato & 3 & $298-453$ & $20,21(18,90-21,05)$ & $5,62(4,49-7,35)$ \\
Etil laurato & 7 & $290-361$ & $4,47(2,44-6,76)$ & $4,03(1,53-6,24)$ \\
Etil miristato & 9 & $293-348$ & $10,47(8,15-12,15)$ & $3,71(1,76-6,39)$ \\
Etil palmitato & 18 & $295-419$ & $17,06(14,09-19,66)$ & $2,35(0,39-5,84)$ \\
Etil estearato & 1 & 348 & 17,79 & 7,59 \\
Etil oleato & 5 & $293-453$ & $19,73(15,29-21,93)$ & $9,37(6,99-15,41)$ \\
\hline
\end{tabular}

\section{Conclusão}

Neste trabalho, um conjunto de métodos para previsão de propriedades de ésteres metílicos e etílicos de cadeia longa (C12-C18) foi selecionado para modelar propriedades de interesse na combustão de biodieseis. Os métodos que se mostraram mais adequados para prever as propriedades de interesse dentre os métodos pesquisados foram: o método de Marrero e Gani para estimar temperatura crítica e pressão crítica; o método de Constantinou e Gani para o volume crítico e o de Han e Peng para o fator acêntrico. $O$ método de Joback modificado pela equação de Bondi foi indicado para a avaliação da capacidade calorífica; o método de Constantinou e Gani para entalpia de vaporização. Os métodos de Joback, Constantinou e Gani; e Marrero-Gani foram equivalentes e apresentam estimativas precisas de entalpia de combustão dos ésteres avaliados. O método de Krisnangkura estimou com confiabilidade a viscosidade dos ésteres quando líquidos em temperaturas de até 422 K. O método de Pitzer e Curl foi indicado para cálculos de tensão superficial com erros inferiores ao método de Brock e Bird o qual havia sido indicado como eficiente na literatura consultada

\section{Agradecimentos}

Sarah Arvelos agradece ao CNPq pela bolsa de mestrado, concedida no período de 20122013. O presente trabalho foi realizado com apoio da Coordenação de Aperfeiçoamento de Pessoal de Nível Superior - Brasil (CAPES) Código de Financiamento 001.

\section{Referências Bibliográficas}

${ }^{1}$ Mahmudul, H. M.; Hagos, F. Y.; Mamat, R.; Adam, A. A.; Ishak, W. F. W.; Alenezi, R. Production, characterization and performance 
of biodiesel as an alternative fuel in diesel engines - A review. Renewable and Sustainable Energy Reviews 2017, 72, 497. [CrossRef].

${ }^{2}$ Gebremariam, S. N.; Marchetti, J. M. Economics of biodiesel production: Review. Energy Conversion and Management 2018, 168, 74. [CrossRef]

${ }^{3}$ Urioste, D.; Castro, M. B. A.; Biaggio, F. C.; Castro, H. F. Síntese de padrões cromatográficos e estabelecimento de método para dosagem da composição de ésteres de ácidos graxos presentes no biodiesel a partir do óleo de babaçu. Química Nova 2008, 31, 407. [CrossRef]

${ }^{4}$ Ramos, L. P.; Kothe, V.; César-Oliveira, M. A. F.; Muniz-Wypych, A. S.; Nakagaki, S.; Krieger, N.; Wypych, F.; Cordeiro, C. S. Biodiesel: Matérias-primas, tecnologias de produção e propriedades combustíveis. Revista Virtual de Química 2017, 9, 317. [CrossRef]

${ }^{5}$ Meneghetti, S. M. P.; Meneghetti, M. R.; Brito, Y. C. A reação de transesterificação, algumas aplicações e obtenção de biodiesel. Revista Virtual Química 2013, 5, 63.[CrossRef]

${ }^{6}$ Koekman, S. K.; Broch, A.; Robbins, C.; Ceniceros, E.; Natarajan, M. Review of biodiesel composition, properties, and specifications. Renewable and Sustainable Energy Reviews 2012, 16, 169. [CrossRef]

${ }^{7}$ Sakthivel, R.; Ramesh, K.; Purnachandran, R.; Shameer, P. M. A review on the properties, performance and emission aspects of the third generation biodiesels. Renewable and Sustainable Energy Reviews 2018, 82, 2970. [CrossRef]

${ }^{8}$ Anand, K.; Sharma, R. P.; Mehta, P. S. A comprehensive approach for estimating thermo-physical properties of biodiesel fuels. Applied Thermal Engineering 2011, 31, 235. [CrossRef]

${ }^{9}$ An, H.; Yang, W. M.; Maghbouli, A.; Chou, S. K.; Chua, K. J. Detailed physical properties prediction of pure methyl esters for biodiesel combustion modeling. Applied Energy 2013, 102, 647. [CrossRef]
${ }^{10}$ Hong, I. K.; Jeon, G. S.; Lee, S. B. Prediction of biodiesel fuel properties from fatty acid alkyl esters. Journal of Industrial and Engineering Chemistry 2014, 20, 2348. [CrossRef]

${ }^{11}$ Saxena, P.; Jawale, S.; Joshipura, M. H. A review on prediction of properties of biodiesel and blends of biodiesel. Procedia Engineering 2013, 51, 395. [CrossRef]

${ }^{12}$ Samavi, M.; Ghobadian, B.; Ardjmand, M.; Seyfkordi, A. Prediction of biodiesel properties and its characterization using fatty acid profiles. Korean Journal of Chemical Engineering 2016, 33, 2042. [CrossRef]

${ }^{13}$ Mostafaei, M. Prediction of biodiesel fuel properties from its fatty acids composition using ANFIS approach. Fuel 2018, 229, 227. [CrossRef]

${ }^{14}$ Gülüm, M.; Onay, F. K.; Bilgin, A. Comparison of viscosity prediction capabilities of regression models and artificial neural networks. Energy 161, 15, 361. [CrossRef]

${ }^{15}$ Yuan, W.; Hansen, A. C.; Zhang, Q. Predicting the physical properties of biodiesel for combustion modeling. Transaction of the ASAE 2003, 46, 1487.[CrossRef]

${ }^{16}$ Ceriani, R.; Gani, R.; Meirelles, A. J. A. Prediction of heat capacities and heats of vaporization of organic liquids by group contribution methods. Fluid Phase Equilibria 2009, 283, 49. [CrossRef]

${ }^{17}$ Do Carmo, F. R.; Sousa, P. M.; SantiagoAguiar, R. S.; De Sant'ana, H. B. Development of a new model for biodiesel viscosity prediction based on the principle of corresponding state. Fuel 2012, 92, 250. [CrossRef]

${ }^{18}$ Pratas, M. J.; Freitas, S. V. D.; Oliveira, M. B.; Monteiro, S. C.; Lima, Á. S.; Coutinho, J. A. P. Densities and Viscosities of Minority Fatty Acid Methyl and Ethyl Esters Present in Biodiesel. Journal of Chemical \& Engineering Data 2011, 56, 2175. [CrossRef]

${ }^{19}$ Kim, S-K.; Choi, H-S.; Kim, Y. Thermodynamic modeling based on a generalized cubic equation of state for 
kerosene/Lox rocket combustion. Combustion and Flame 2012, 159, 1351. [CrossRef]

${ }^{20}$ Lopez-Echeverry, J. S.; Reif-Acherman, S.; Araujo-Lopez, E. Peng-Robinson equation of state: 40 years through cubics. Fluid Phase Equilibria 2017, 15, 39. [CrossRef]

${ }^{21}$ Tesfa, B.; Gu, F.; Mishra, R.; Ball, A. D. LHV predication models and LHV effect on the performance of $\mathrm{Cl}$ engine running with biodiesel blends. Energy Conversion and Management 2013, 71, 217. [CrossRef]

${ }^{22}$ Aminian, A.; ZareNezhad, B. Accurate predicting the viscosity of biodiesels and blends using soft computing models. Renewable Energy 2018, 120, 488. [CrossRef]

${ }^{23}$ Freitas, S. V. D.; Oliveira, M. B.; Queimada, A. J.; Pratas, M. J.; Lima, A. S.; Coutinho, J. A. P. Measurement and prediction of biodiesel surface tensions. Energy \& Fuels 2011, 25, 4811. [CrossRef]

${ }^{24}$ Joback, K. G.; Reid, R. C. Estimation of PureComponent Properties from GroupContributions. Chemical Engineering Communications 1987, 57, 233. [CrossRef]

${ }^{25}$ Constantinou, L.; Gani, R.; O'Connell, J. P. Estimation of the acentric factor and the liquid molar volume at $298 \mathrm{~K}$ using a new group contribution method. Fluid Phase Equilibria 1995, 103, 11. [CrossRef]

${ }^{26}$ Marrero-Morejón, J.; Pardillo-Fontdevila, E. Estimation of pure compound properties using group-interaction contributions. AIChE Journal 1999, 45, 615. [CrossRef]

${ }^{27}$ Marrero, J.; Gani, R. Group-contribution based estimation of pure component properties. Fluid Phase Equilibria 2001, 183184, 183. [CrossRef] [PubMed]

${ }^{28}$ Poling, B. E.; Prausnitz, J. M.; O'Connell, J. P.; The Properties of Gases and Liquids, 5th. ed, McGraw-Hill: New York, 2000. [Link]

${ }^{29}$ Kesler, M. G.; Lee, B. I. Improve Predictions of Enthalpy. Hydrocarbon Processing 1976, 55, 153. [Link]

${ }^{30}$ Edmister, W. C. Applied hydrocarbon thermodynamic, part 4: compressibility factors and equation of state. Petroleum
Refiner 1958, 37, 173. [Link]

${ }^{31}$ Han, B.; Peng, D. A group contribution correlation for predicting the acentric factors of organic compounds. The Canadian Journal of Chemical Engineering 1993, 71, 332. [CrossRef]

${ }^{32}$ Rihani, D. N.; Doraiswamy, L. K. Estimation of heat capacity of organic compounds from group contributions. Industrial \& Engineering Chemistry Fundamentals 1965, 4, 17. [CrossRef] [PubMed]

${ }^{33}$ Bondi, A. Estimation of the heat capacity of liquids. Industrial \& Engineering Chemistry Fundamentals 1966, 5, 442. [CrossRef]

${ }^{34}$ Krisnangkura, K. Estimation of heat of combustion of triglycerides and fatty acid methyl esters. Journal of the American Oil Chemists' Society 1991, 68, 56. [CrossRef]

${ }^{35}$ Graboski, M. S.; McCormick, R. L. Combustion of fat and vegetable oil derived fuels in diesel engines. Progress in Energy Combustion Science 1998, 24, 125. [CrossRef]

${ }^{36}$ Santander, C. M. G.; Rueda, S. M. G.; Da Silva, N. D. L.; De Camargo, C. L.; Kieckbusch, T. G.; Maclel, M. R. W. Measurements of normal boiling points of fatty acid ethyl esters and triacylglycerols by thermogravimetric analysis. Fuel 2012, 92, 158. [CrossRef]

${ }^{37}$ Evangelista, N. S.; Carmo, F. R.; Sant'Ana, H. B. Estimation of physical constants of biodiesel-related fatty acid akyl esters: normal boiling point, critical temperature, critical pressure, and acentric factor. Industrial \& Engineering Chemistry Research 2018, 57, 8552. [CrossRef]

${ }^{38}$ Rose, A.; Supina, W. R. Vapor Pressure and Vapor-Liquid Equilibrium Data for Methyl Esters of the Common Saturated Normal Fatty Acids. Journal of Chemical \& Engineering Data 1961, 6, 173. [CrossRef]

${ }^{39}$ Pratas, M. J.; Freitas, S.; Oliveira, M. B.; Monteiro, S. C.; Lima, A. S.; Coutinho, J. A. P. Densities and viscosities of fatty acid methyl and ethyl esters. Journal of Chemical \& Engineering Data 2010, 55, 3983. [CrossRef]

${ }^{40}$ Scott, T. A.; Macmillan, D.; Melvin, E. H. Vapor Pressures and Distillation of Methyl 
Esters of Some Fatty Acids. Industrial \& Engineering Chemistry Fundamentals 1952, 44, 172. [CrossRef]

${ }^{41}$ McCutcheon, J. W. Linoleic acid and its isomers. Canadian Journal of Research 1938, 16, 158. [CrossRef]

42 McCutcheon, J. W. Linolenic acid and its isomers. Canadian Journal of Research 1940, 18, 231. [CrossRef]

${ }^{43}$ Shigley, J. W.; Bonhorst, C. W.; Liang, P. M.; Althouse, P. M.; Triebold, H. O. Physical characterization of a) a series of ethyl esters and b) a series of ethanoate esters. Journal of the American Oil Chemists' Society 1955, 32, 213. [CrossRef]

${ }^{44}$ Omar, M. M.; Davies, E. Vapour pressures, heats of sublimation, and heats of vaporisation for straight-chain ethyl esters. Journal of the Chemical Society C Organic 1967, 2038. [CrossRef]

${ }^{45}$ Silva, L. Y. A.; Falleiro, R. M. M.; Meirelles, A. J. A.; Krähenbühl, M. A. Determination of the vapor pressure of ethyl esters by Differential Scanning Calorimetry. The Journal of Chemical Thermodynamics 2011, 43, 943. [CrossRef]

46 Peng, D.-Y.; Robinson, D. B. A New TwoConstant Equation of State. Industrial \& Engineering Chemistry Fundamentals 1976, 15, 59. [CrossRef] [PubMed]

47 Thomson, G. H. The DIPPR databases. International Journal of Thermophysics 1996, 17, 223. [CrossRef]

48 Nikitin, D.; Popov, A. P. Vapour-liquid critical properties for compounds of biodiesel. 1. Methyl esters of n-alkanoic acids. Fuel 2015, 153, 634. [CrossRef]

${ }^{49}$ Van Bommel, M. J.; Oonk, H. A. J.; Van Miltenburg, J. C. Heat capacity measurements of 13 methyl esters of $n$-carboxylic acids from methyl octanoate to methyl eicosanoate between $5 \mathrm{~K}$ and $350 \mathrm{~K}$. Journal of Chemical \& Engineering Data 2004, 49, 1036. [CrossRef]

${ }^{50}$ Ceriani, R.; Gani, R.; Meirelles, A. J. A.Prediction of heat capacities and heats of vaporization of organic liquids by group contribution methods. Fluid Phase Equilibria 2009, 283, 49. [CrossRef]

${ }^{51}$ Fuchs, R.; Peacock, L. A. Heats of vaporization of esters by the gas chromatography-calorimetry method. Canadian Journal of Chemistry 1980, 58, 2796. [CrossRef]

52 Lipkind, D.; Kapustin, Y.; Umnahanant, P.; Chickos, J. S. The vaporization enthalpies and vapor pressures of a series of unsaturated fatty acid methyl esters by correlation gas chromatography. Thermochimica Acta 2007, 456, 94. [CrossRef]

${ }^{53}$ Freedman, B.; Bagby, M. O. Heats of combustion of fatty esters and triglycerides. Journal of the American Oil Chemists' Society 1989, 66, 1601. [CrossRef]

${ }^{54}$ Mehta, P. S.; Anand, K. Estimation of a lower heating value of vegetable oil and biodiesel fuel. Energy and Fuels 2009, 23, 3893. [CrossRef] 